\title{
NUMERICAL INVESTIGATION OF PUMPING EFFECTS IN FPW-DEVICES
}

\author{
Nam Trung Nguyen*, Roger W. Doering and Richard M. White \\ Berkeley Sensor \& Actuator Center, Department of Electrical Engineering and Computer Science \\ University of California Berkeley \\ Berkeley CA 94720
}

\begin{abstract}
In this paper, we will present the numerical investigation of acoustically driven flow in micromachined flexural plate wave pumps using computational fluid dynamics (CFD). The simulation uses moving grids and moving wall models of a commercially available CFD tool (CFDRC Corporation). The simulation is carried out for a two-dimensional simple channel structure. The results will be discussed at the end of this paper.
\end{abstract}

\section{INTRODUCTION}

Recently there has been considerable interest on the use of flexural plate wave (FPW) devices for pumping fluid [1,2]. The pumping principle is based on the phenomenon of acoustic streaming in which high intensity acoustic fields cause the fluid flow. The acoustic field occurs near a surface on which flexural plate waves propagate. This pumping principle enables the realization of an "active" micro channel, which overcomes the high impedance problem encountered during the delivery of fluids through micro channels.

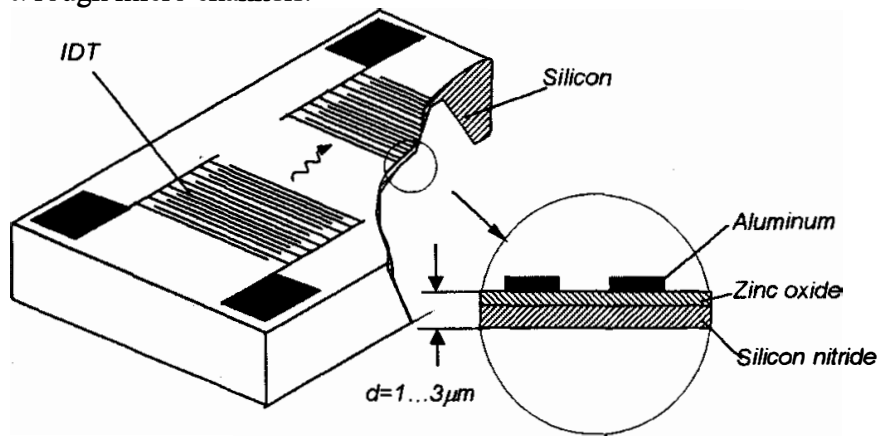

Figure 1. Classical flexural plate wave device

In our previous work [3], an analytical theory was presented, which has demonstrated that acoustic streaming in the fluid is responsible for the pumping effect. Recently a numerical study of flow through wavy-walled channels that is similar to our case was reported [4]. However, this work only studied the first-order velocity field. Our paper presents the numerical results of timeaveraged velocity field that causes acoustic streaming.

The numerical model is based on pumping water with a basic FPW-device shown in Fig. 1. The device consists of a rectangular flow channel that has a thin membrane on the bottom. The membrane is made of piezoelectric zinc oxide, aluminum, and lowstress silicon nitride. The typical thickness of the membrane measures from 1 to 3 microns. The flexural plate waves have a typical frequency of $3 \mathrm{MHz}$ and a wavelength of 100 microns. Interdigitated transducers (IDTs), that are arrays of finger pairs placed at wavelength intervals, generate the flexural plate waves.

\section{FORMULATION OF THE NUMERICAL MODEL}

Fig. 2 describes the two-dimensional model of the FPWdevice. The channel model has a length of three wavelengths $(3 \lambda=300 \mu \mathrm{m})$. Wave amplitude and channel height are $10 \mathrm{~nm}$ and

\footnotetext{
* Poster presenter
}

$50 \mu \mathrm{m}$ respectively. The model contains a moving wall as the flexural surface, a rigid wall as the opposite surface and two cyclic boundaries as inlet and outlet. The cyclic boundary condition forces all variables (e. g., velocity, pressure) at the inlet and outlet to be equal. Fig. 2 shows how points on the device surface move elliptically in a counterclockwise direction as the flexural wave travels from left to right.

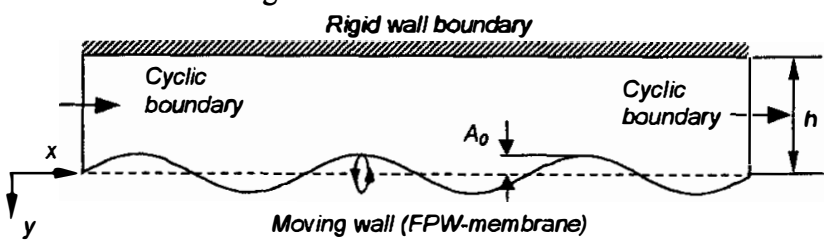

Figure 2. Model of FPW-pump devices

The vertical displacement $\Delta y$ of the plate is given by:

$\Delta y=A_{0} \sin (\omega t-k x)$,

where $A_{0}$ is the wave amplitude, $\omega=2 \pi f$ is the angular frequency of the wave, and $k=2 \pi / \lambda$ is the wave number. With a membrane thickness $d$, the horizontal displacement is [5]:

$\Delta x=\left(A_{0} \pi d / \lambda\right) \cos (\omega t-k x)$.

Thus the ratio of the minor to the major axis of the ellipse is given by $\pi d / \lambda$. For instance, with the wavelength of $\lambda=100 \mu \mathrm{m}$ and the membrane thickness of $d=3 \mu \mathrm{m}$, the ratio of the minor to major axis of the elliptical motion will be 0.093 .

From Eqs. (1) and (2) the velocity boundary conditions for the moving wall are:

$u_{w}=d(\Delta x) / d t=-2 \pi^{2}\left(A_{0} d f / \lambda\right) \sin (\omega t-k x)$
$v_{w}=d(\Delta y) / d t=2 \pi f A_{0} \cos (\omega t-k x)$

Since the CFD program implements both the moving wall and moving grid, there are two ways for building flexural plate wave boundary conditions: updating grid coordinates in the whole model or updating wall velocity components on the moving wall.

Using the first strategy, we assume that the computational grid moves elliptically. The grid position is updated for every time step using equations (1) and (2). For the second case we assume that the wave amplitude is much smaller than channel height $h$ (several tens of nanometers versus several tens or hundreds of microns). The grids don't move; only the velocities of the moving wall are updated for every time step using Eqs. (3) and (4). The results presented in this paper were obtained with the first strategy.

A user library routine written in FORTRAN modifies the grid positions and velocity boundary conditions. Essential parameters of the model, and the current time and grid position, can be provided from pre-defined user constants and user routines of the software package. In order to make a realistic initial condition, the wave amplitude is described as a function of time:

$A_{0}(t)=A_{\max }(1-\exp (-t / \tau))$

For the results in this paper, we assume a time constant $\tau$ for water of one wave period $T=1 / f$. The simulation extends over more then 15 periods with 10 time steps each. The pressure field and the first-order velocity field of every time step are saved and used as the initial condition for the next time step. The time-averaged 
velocity field can be than calculated and depicted by using MATLAB.

\section{RESULTS}

Fig. 4(a) illustrates the evolution of the pressure distribution in a one-wavelength portion of the channel over one time period. The mesh plot represents the pressure values of the last contour plot. Fig. 4(a) shows clearly that the acoustic field is evanescent with a decay length of about $20 \mu \mathrm{m}$.

Fig. 4(b) shows the time-dependent velocity field of the same region shown in Fig. 4(a). Using this field, the time-averaged velocity field shown in Fig. 4(c) can then be calculated. This field is responsible for the pumping effect. A shear viscous field penetration depth of about $5 \mu \mathrm{m}$ can be observed in Fig. 5. The maximum time-averaged velocity is $2.2 \mathrm{~mm} / \mathrm{s}$.

\section{CONCLUSIONS}

We have demonstrated the numerical simulation of the pumping effect in flexural plate wave devices using commercial CFD tools. However, the simulation is very time consuming. For a model with 3240 elements and 150 time steps (15 periods), the results presented in this paper took about 24 hours running on 200 MHz Pentium-PC with Windows NT. An optimization considering the number of grids, time step and time constant of the wave amplitude function could be done in order to get a faster simulation with acceptable accuracy. Most importantly the results reported in this paper show that the systematic design and optimization of acoustic micropumps is possible.

\section{ACKNOWLEDGEMENTS}

The authors would like to thank Dr. A. Lal of the University of Wisconsin-Madison and the staff of CFDRC Corporation for the initial work on this subject and their assistance.

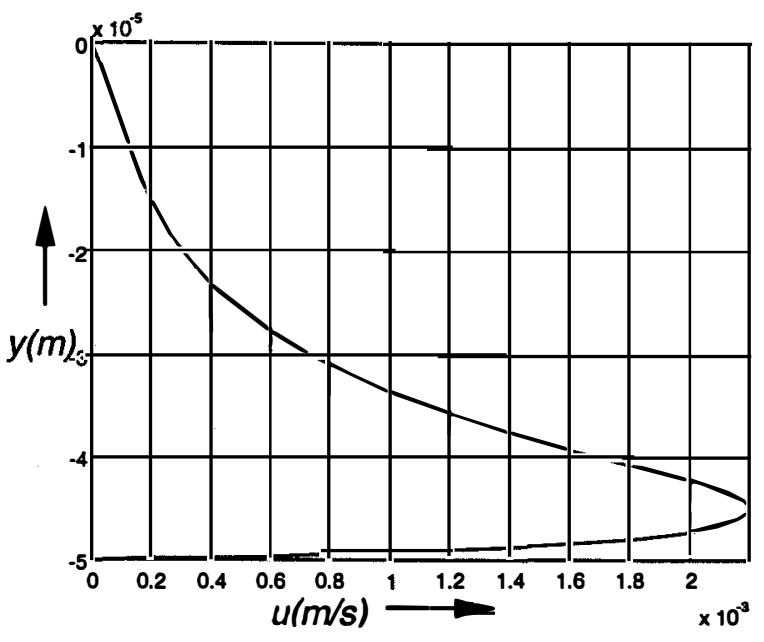

Figure 5. Profile of the time-averaged velocity over the channel height (velocity component in $x$-direction)

\section{REFERENCES}

1. R. M. Moroney, R. M. White, R. T. Howe, "Microtransport induced by ultrasonic Lamb waves", Applied Physics Letters, 59,7(1991), pp. 774-776.

2. P. Luginbuhl, S. D. Collins, G. A. Racine, M. A. Gre'tillat, N. F. de Rooij, K. G. Brooks and N. Setter, "Microfabricated Lamb wave device based on PZT sol-gel thin film for mechanical transport of solid particles and liquids", Journal of Microelectromechanical Systems, 6, 4(1997), pp. 337-346.

3. C. E. Bradley, R. M. White, "Acoustically driven flow in flexural plate wave devices: Theory and experiments" Proc. IEEE Ultrasonics Symp. (1994), pp. 593-597.

4. S. Selvarajan, E. G. Tulapurkara and Vasanta Ram, "A numerical study of flow through wavy-walled channels", International Journal for Numerical Methods in Fluids, 26(1998), pp. 519-531.

5. T. Sashida and T. Kenjio, "An instroduction to Ultrasonic Motors”, Oxford: Clarendon Press, 1993, pp. 130.

a)

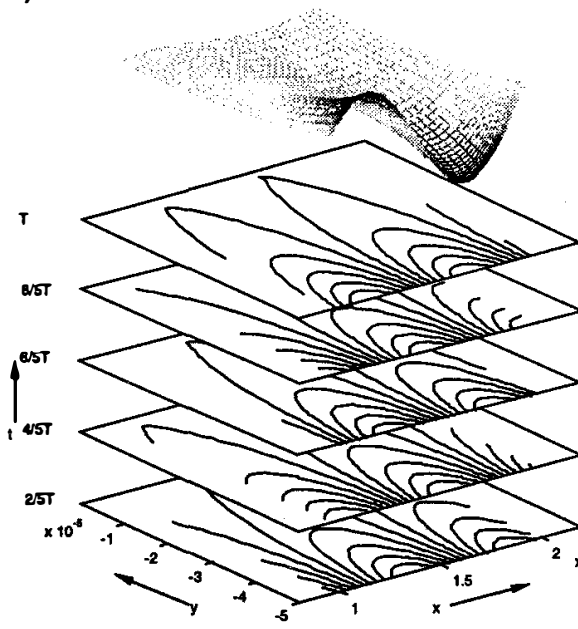

b)

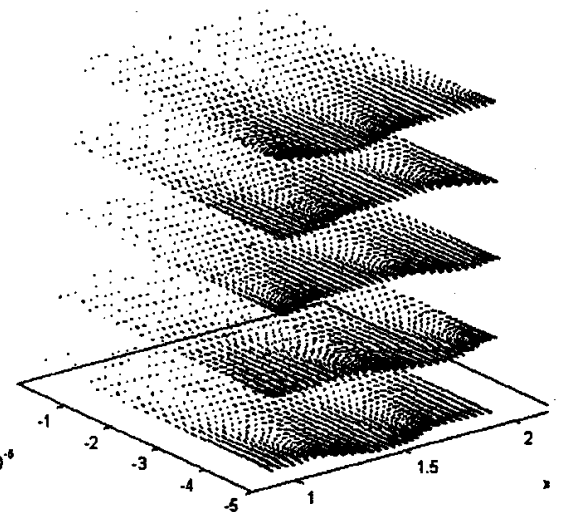

c)

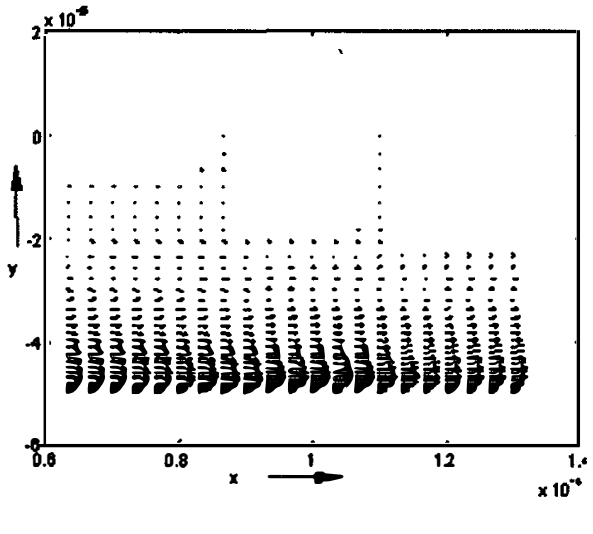

Figure 4. Simulation results for the micromachined FPW-pump. (a) The pressure fields over a time period T (propagation is in the $x$ direction). (b) The velocity fields corresponding to the pressure fields shown in (a). (c) The time-averaged velocity field in the channel resulting from the time-dependent velocity fields in $(b)$. 